\title{
Twenty Years of Research on Cholesteric Lyotropic Liquid Crystals at the Chemistry Institute of the University of São Paulo
}

\author{
M. R. Alcantara and E. G. Fernandes Jr. \\ Instituto de Química, Universidade de São Paulo, \\ Av. Prof. Lineu Prestes 748, Cidade Universitária, \\ CEP 05508-900, São Paulo, SP, Brazil.
}

Received on 25 November, 2001

\begin{abstract}
In this paper we present a review of twenty years of research on cholesteric liquid crystals at the Chemistry Institute of the University of São Paulo. The results on the interaction forces, responsible for the maintenance of the helical structure, are a function of the chemical variability obtained in these systems. The last results obtained using rheological techniques are presented, and research in this field was undertaken in order to understand the cholesterization process when different systems and inductors are involved.
\end{abstract}

\section{Introduction}

Any kind of review concerning the field of Liquid Crystals should begin with the work of Reinitzer [1] and Lehmann [2], when this class of materials was discovered. However, the results obtained, by the end of the XIX century, on the fusion of cholesteryl benzoate and its birefringence observed by means of polarized microscope, had no impact on the scientific community.

Friedel [3], in his classical work dated 1922, studied several mesomorphic systems observed via the polarized microscope, classifying them through their observed textures and properties. He was the first scientist to systematize the knowledge on Liquid Crystals, creating a complete database introducing the nomenclature still in use.

Later, in 1933, the Faraday Society promoted a discussion about the activities developed during the first three decades of studies on liquid crystals. It may be observed that impressive names as Bernal, Lawrence and Zocher [4] registered their results during this meeting. It is also possible to verify that, by that time, the field of Lyotropic Liquid Crystals was still almost completely ignored.

After the Faraday Society Meeting, the subject "Liquid Crystals" fell into a period of little activity. The development of new experimental techniques and new materials would be vital to promote further improvement. During this period, the work of McBain [58], clarifying several phase diagrams of binary and ternary systems based on amphiphile and water, should be noted.
In 1962, Saupe \& Englert [9] observed for the first time the NMR proton spectrum of benzene oriented on a Liquid Crystal. Flautt and Lawson [10] made the great step in lyotropic investigation with the observation, by ${ }^{1} \mathrm{H}$ NMR, of the broad line of a quaternary system based on sodium decylsuphate. At the same time, Prof. Leonard W. Reeves, professor at the Waterloo University, Canada, accepted an invitation to start, at the Chemistry Institute of the University of São Paulo, a new branch of research inserted in the context of "modern Physic-Chemistry". It was the beginning of the research of Liquid Crystals in Brazil.

The investigation into lyotropic liquid crystals started with the study of the structure of molecules and ions dissolved in lyomesomorphic systems but, after 1970, the lyotropic micelle became one of the main target of study in this field, with NMR, especially the ${ }^{2} \mathrm{H}$ NMR, being the main technique used for this purpose. The studies of Reeves [11, 12] and Charvolin [13, 14], during this time, should be emphasized. A basic knowledge about the techniques for order profile analysis of perdeuterated chains was obtained during this time, with the basis of the work being developed by Seeling $[15,16]$ and Smith $[17,18]$, in their study on biologic membrane structure.

In 1975, Diehl \& Tracey [19] obtained, for the first time, an induced cholesteric lyotropic system with the addition of sodium 2-decylsuphate to a lyomesophase based on sodium decylsulphate. However, in this work, the main objective was not the cholesteric properties of the system, but the distinction between two optical isomers of the racemic solute, using NMR. 
It was only in 1978 that Radley \& Saupe [20] published systematic studies about induced cholestericity on Lyotropic Liquid Crystals. The investigation of cholesteric systems, obtained by the addition of cholesterol, tartaric acid or brucine sulphate, was pioneered by Saupe and co-workers. It was the beginning of the activities of Saupe in the investigation into lyotropic liquid crystals. These authors demonstrated that these mesophases showed, after an orientation by a magnetic field, observed by means of the polarized microscope, textures similar to those observed for PBLG (poly- $\gamma$ benzyl-L-glutamate).

The year of 1980 should be considered as a reference in the field of cholesteric lyotropic crystals, because it was in this year that new cholesteric lyomesomorphic systems, using chiral amphiphiles [21], were prepared for the first time. The term Intrisic Cholesteric was given to these systems since their micelles were intrinsically chiral. It was in the same year that J.A. Vanin started the study of these systems at the Chemistry Institute of the University of São Paulo. Several works using these phases could be developed here in Brazil [22-28]. Several chiral amphiphiles, derived from $\mathrm{N}$-acyl-amino acids, were synthesized leading to several intrinsic cholesteric systems that had their optical and magnetic properties extensively studied [23-25].

The use of chiral amphiphiles, as precursors of cholesteric lyomesophases, was responsible for the increase in the number of systems as well as for revealing the possible anomalies resulting from their cholesteric properties. The chemical variability obtained in these new systems would be responsible for a new branch of research, mainly concerned with the study of intermicellar interactions. Several research projects comparing the properties obtained for these intrinsic cholesteric systems, with those obtained for induced cholesteric ones, were developed over the following years.

The distortion of the helicoidal arrangement due to the competition between the wall effect orientation and the twist power of different inductors, was verified through optical microscopy utilizing polarized light techniques [26]. The helical twist sense of different induced cholesteric lyophases was determined and it was possible to observe that the absolute configuration of the inductor is important only when the inductor is located predominantly in the aqueous portion [28]. It was possible to verify, also, that the twist sense does not exclusively depend on the absolute configuration of the chiral molecule. When the inductor is located predominantly in the aqueous portion its structure (that comprises constituition, configuration and conformation) is solely responsible for the helical twist sense. A hydrophobic inductor, even when submitted to structural changes due to strong interactions in the hydrocarbon core, should induce the same helical twist sense for different lyomesophases. Finally, for inductors submitted to strong electrical double layer interactions, the twist sense should be determined by an inductor/micelle interaction [28].

Since the beginning of the studies on cholesteric lyotropic liquid crystals at the Chemistry Institute of the University of São Paulo, the characterization of cholesteric systems was revealed to be simple in terms of their basic properties, but extremely complex when it came to a complete understanding of the fundamental interactions responsible for the formation of the helical arrangement. During several years of work it was impossible to identify different cholesterization mechanisms in relation to phase type and on the nature of its components [25-28]. In 1995, the search for new techniques that could contribute to the understanding of the forces acting on these association colloids led us to Rheology.

The rheologic behavior of a system evaluates its resistance to flow and to deformation, allowing the estimation of a macroscopic mean value for the structural and hydrodynamic interaction between its constituent particles, this being dependent on parameters like density, stability, molecular mass and particle dimensions [29-31]. In recent years, the rheologic properties of many materials were used by several groups [32$45]$ in order to gain information about the spatial arrangement of the units that constitute the system, and its changes in the phase transition regions [32, 33].

The theoretical studies on liquid crystals rheology began with Ericksen [46] and Leslie [47, 48], who proposed the flow description of nematic and cholesteric systems using the continuum theory [48]. Although some reports exist on the dynamic properties of liquid crystals [49], all of them were limited to parallel plates, concentric cylinders or capillary flow geometries. The studies on viscosity, using cone-and-plate geometry, showed complicated results since the director's orientation is not defined at the instant of the measurement. The problem is particularly complicated when using large volumes of samples, since the viscometer walls [47] can cause orientation and disclination effects [49]. Some efforts have been made to determine the arrangement by optical microscopy under polarized light, as well as using rheological techniques [32, 33, 50].

Rey $[51,52]$ developed a theoretical approach to the rheological behavior of cholesteric liquid crystal systems. This approach gave important information on the position and behavior of the helix under the flow. Rey [51, 52] proposed that if the helix was aligned with the flow direction, it would untwist and maintain a configuration similar to that obtained for nematic systems.

Shear-induced changes in structure and orientation in lyotropic liquid crystalline phases of surfactant solutions have met increasing interest over the past few years. The rheological properties of lyotropic liquid crystals are often affected by their mechanical history, which may result in a modification in the microscopic structure of the material. Cooperative changes in the 
conformation of the flexible layer were associated with the flow imposed by the shear on lyotropic liquid crystals $[35,54,55]$. It was possible to observe that, for low shear rate, the lamellae orientation is parallel to the flow direction while, for high shear rate, the orientation of the lamellae becomes perpendicular [53]. Shearinduced transitions were also observed in concentrated solutions at defined has been done on flexible elongated surfactant micelles that can break and recombine continuously as a function of time, being considered as examples of 'living' polymers [56-60].

Results published by our laboratory in this new field showed that ternary systems, based on sodium decylsulphate / sodium sulphate and water, were characterized by their rheopectic behavior (Fig. 1). For up curves only, alternation between a dilatant and a pseudoplastic component was observed according to the shear rate value [34]. The dilatant/pseudoplastic interchange characterizes the occurrence of structural viscosity, usually attributable to a localized increase in particle concentration, or to progressively greater alignment rates, due to competition between the flow velocity gradient and the disordering effect of Brownian motion. In both cases, the observed behavior can be assigned to a superstructure breakdown, arising from shear flow. It could also be observed too that, as a consequence of the sample reorientation process, the dilatant/pseudoplastic interchange is more pronounced when the time interval between successive rheograms is increased [34].

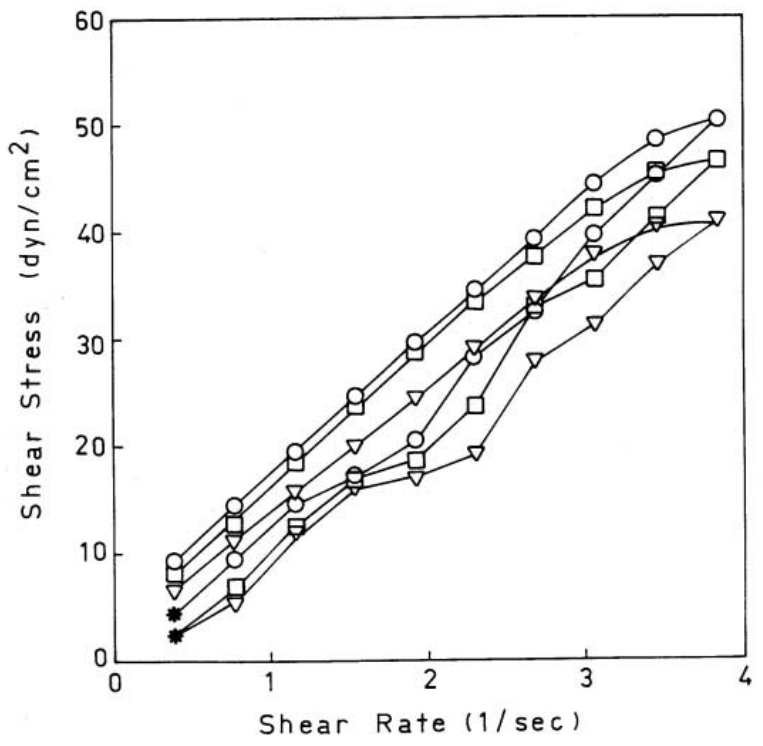

Figure 1. Sequence of rheograms of the $N_{C}$ mesophase repeated at time intervals of : (o) initial rheogram; ( $\square$ ) 6 min; ( $\nabla) 23 \min [34]$.

$N_{D}$ systems, based on sodium decylsulphate, sodium sulphate, n-decanol and water, also presented a pseudoplastic rheopectic behavior [34]. A recovery time can be clearly observed, since consecutive sequences are not superimposable (Fig. 2), as an effect of an orienta- tional competition between flow effects and intermicellar interactions acting in different directions [34].

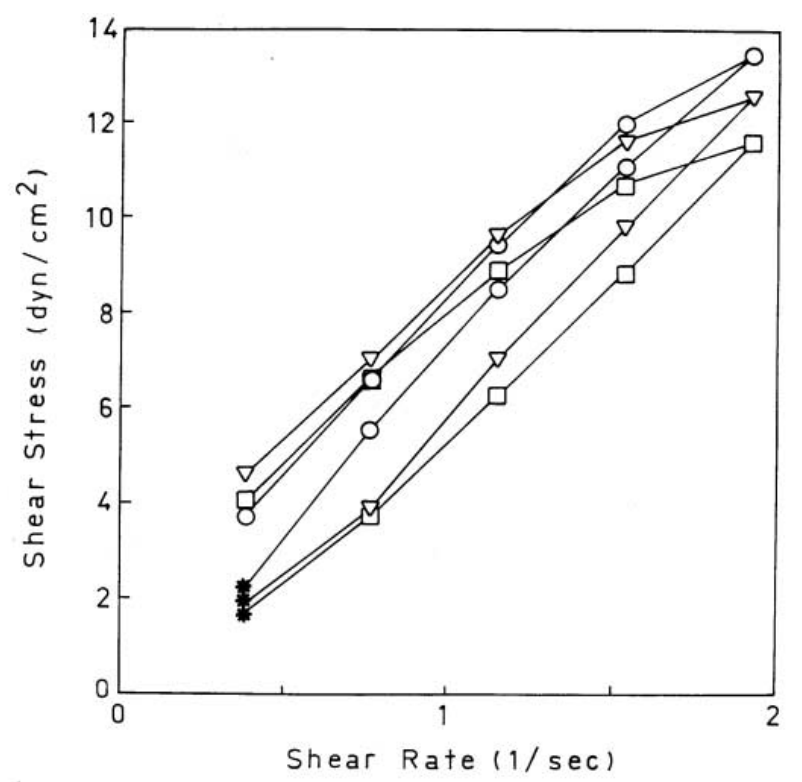

Figure 2. Sequence of rheograms of the $N_{D}$ mesophase repeated at time intervals of : (o) initial rheogram; ( $\square$ ) 9 min; ( $\nabla) 12 \min [34]$.

Studies developed on $N_{C}$ systems, based on dodecylsulphate, showed that when the sample was sheared in an atmosphere without water vapor saturation, an increase in the apparent viscosity was observed. On the other hand, the rheograms, obtained on a saturated atmosphere, showed a decrease in the viscosity [61]. The same system, cholesterized by different amounts of cholesterol, showed a tendency to align the micellar direction parallel to the flow direction [62]. In this sense, the more ordered the system, smaller are the resulting shear stresses. It was also possible to observe that, for cholesteric systems, this orientation process is minimized by the existence of chiral interaction, which precludes the complete untwisting of the helix. It was verified that the higher the inductor concentration, the larger the shear stresses obtained, indicating an increase in the micellar interaction forces. The yield stress remains approximately the same, but the increase in the plastic behavior can be observed as a function of the cholesterol concentration. It was also observed that increasing cholestericity leads to an increase in the consistency index, indicating that the system that is more resistant to deformations, i.e, a helicodal array that is more rigid due to increased chiral interaction forces [62].

The study of water activity behavior in liquid crystals was reported for lyomesophase based on sodium dodecylsulphate (SLS), decylammonium chloride (DAC) and potassium laurate (KL) [63]. The results obtained for these systems showed that SLS mesophase has more tightly bound water molecules after the formation of the helical structure. This result is independent of the 
amount of added solute, showing that, after the initial cholesterization of the system, the micelle-solvent interaction does not increase with the helical structure formation [63].

The system based on DAC shows a reduction in the interaction forces between the micelles and the solvent after the formation of the helical structure. Rheological data showed that, the greater the inductor concentration, the lesser the interactions between micelles in the system. [63].

The system based on KL showed a decrease in the water activity only if hydrophilic inductors were added, possibly because of their higher concentration. No difference due to the cholesterization itself was observed. It was observed that the KL system has a loss of water rate greater than that of the DAC system. However, both systems have high loss rates, making it mandatory to carry out rheological experiments with an adequate control of humidity [63]. It could be concluded that each system has an unique behavior in relation to water activity, due, mainly, to differences in micelle geometry and intermicellar arrangement, but also to the fact that they are constituted by different amphiphiles that, in different ways, interact with the water molecules and with the free ions in the solution.

The occurrence of edge fracture on lyotropic liquid crystals during rheological measurements could be well described for systems based on KL [64]. The results obtained showed that the rheological behavior during the initial shearing (before any fracture took place) may be described as nearly Newtonian, although a thixotropic response causes a small separation between the first and the second shearing and between the third and the fourth shearing. The thixopropic response indicates that the samples contain microdomains, with distinct orientations, that are broken down into a single domain as the shearing proceeds [64]. It could be verified too that a temperature lowering causes the increase of both the elastic and the viscous responses of the system, which may lead to the conditions necessary for the occurence of the edge fracture during shearing. Successive rheograms, performed at different temperatures, (Fig. 3) showed that the fracture first happened the fifth rheogram, where the decreasing of the shear stress to $>70 \mathrm{~s}^{-1}$ could be observed. The sixth rheogram, performed at the same temperature as the fifth, showed an additional decrease of the stress readings after the edge fracture, indicating a further propagation. At each new temperature, a decrease in the fracture shear rate was observed, indicating that the sample fracture increased to a greater extent than that previously observed extent, i.e, the fracture orientation did not relax between any two rheograms. The fracture is stable during the time required for an ordinary rheological measurement, unless the temperature is raised [64]. The amount of shear rate increment along a rheogram determines how sensitive the fracture behavior of the sample to its pre- vious mechanical history would be, it being more sensitive the smaller the size of the increment. It was possible to reorientate the sample and to repeatedly perform the fracture procedure on the same sample, showing that the fracture threshold shear rate increases as the sample becomes more aligned [64].

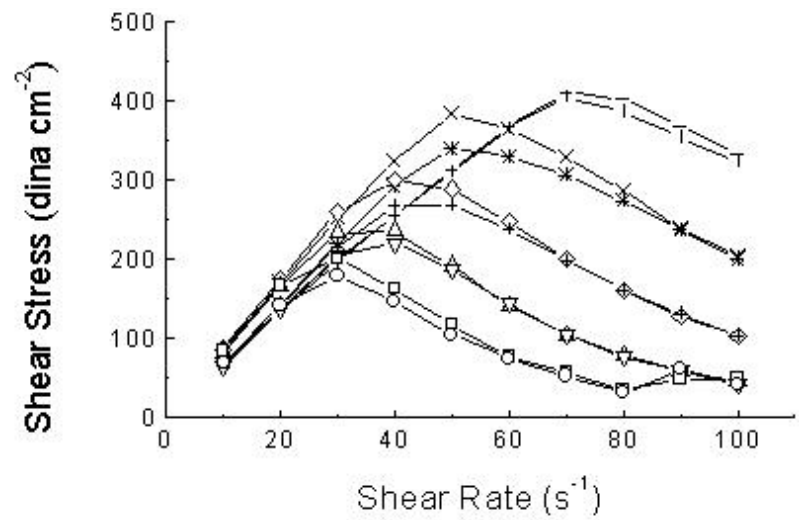

Figure 3. Rheograms of the $\mathrm{LK} / \mathrm{KCl} / \mathrm{DeOH} / \mathrm{H}_{2} \mathrm{O}$ system : $-5^{t h}$ shearing $\left(25.0^{\circ} \mathrm{C}\right) ;-1-6^{t h}$ shearing $\left(25.0^{\circ} \mathrm{C}\right) ;-*_{-} 8^{t h}$ shearing $\left(22.5^{\circ} \mathrm{C}\right) ;-\diamond-9^{t h}$ shearing $\left(20.0^{\circ} \mathrm{C}\right) ;-+-10^{t h}$ shearing $\left(20.0^{\circ} \mathrm{C}\right) ;-\triangle-11^{\text {th }}$ shearing $\left(17.5^{\circ} \mathrm{C}\right) ;-\nabla-12^{\text {th }}$ shearing $\left(17.5^{\circ} \mathrm{C}\right) ;-\square-13^{\text {th }}$ shearing $\left(15.0^{\circ} \mathrm{C}\right) ;-0-14^{\text {th }}$ shearing $\left(15.0^{\circ} \mathrm{C}\right)[64]$.

The threshold shear rate for the edge fracture to occur depends upon the type and degree of the sample alignment. Nematic lyotropic liquid crystals have long-range orientational order. The original structure was formed by several microdomains and the region between any two microdomains with different orientation may be viewed as a structural defect. When the sample is sheared, a local stress increase takes place around each of these defects [64]. Part of this stress excess is spent in the form of a plastic work, aligning the micelles, while the rest of it may initiate a fracture. A compromise between the initiation and the propagation steps was observed. The presence of defects is necessary for the fracture to be initiated but a great number of them in the original sample did not contribute to decreasing the fracture shear rate [64].

The rheological behavior of cholesteric lyotropic liquid crystals can give information about the chiral forces involved on the formation of the cholesteric arrangement. The effect of these forces on the orientation process of a liquid crystal based on DAC and submitted to a period of shear was studied [65]. The results obtained showed that the cholesteric system acquires an initial pseudoplastic arrangement that turns to Newtonian as a function of the rest time or the shear time (Fig. 4). The orientation process should involve an initial step involving the destruction of the cholesteric structure caused by shear, leading to the formation of microdomains with different orientations. In a second stage these domains will be orientated either by a shear process or by the performance of the elastic constants 
forming the final structure that is quite similar to the nematic one [65].

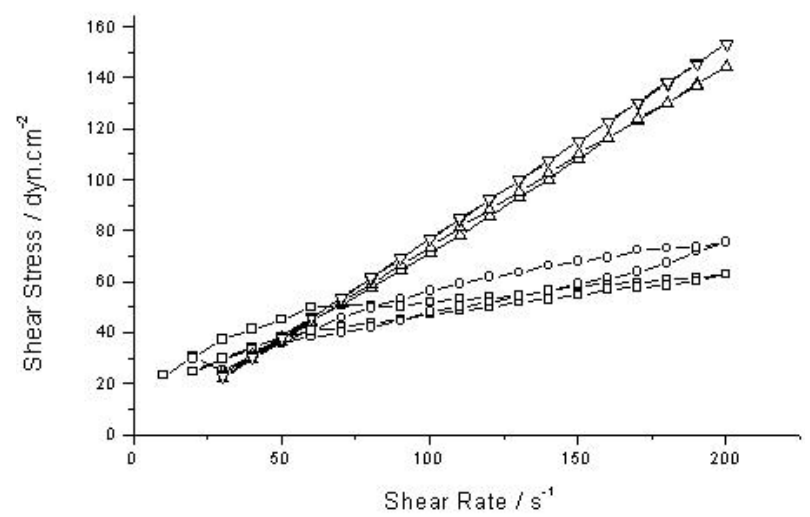

Figure 4. Rheograms for the cholesteric phase with $0.09 \%$ molar of cholesterol (CDA10): ( $\square) 1^{\text {st }}$ sweep; (o) $2^{\text {nd }}$ sweep (immediately after first); $(\triangle) 10 \mathrm{~min}$ after $2^{\text {nd }} ;(\nabla) 30 \mathrm{~min}$ after $3^{\text {rd }}[65]$.

The same DAC system was also studied as a function of the kind of inductor present in its composition. The results obtained showed a change in the rheological behavior dependent on the chiral inductor nature. Phases with hydrophobic inductor showed a viscosity decrease with an increase in cholesterol concentration [66], while an increase in D-(+)-Mannose concentration leads to a viscosity increase [67].

Changes in the rheological behavior were also observed according to the amount of solvent present in the phase. For systems with cholesterol and with a greated amount of water, the viscosity increases with the time until reaching a maximum value, followed by a decrease. This behavior indicates that the phases should suffer some kind of deformation before reaching their maximum tensions. After that, an accommodation process of the structure causes a viscosity decrease [66]. For the systems with hydrophobic inductors, and with a lesser amount of solvent, the viscosity decreases to a minimum value, which is reached later as a function of increasing inductor concentration. After that, there is an increase in the viscosity until a constant value is obtained. This suggests the occurrence of a breakdown of the structure, followed by its reorientation by the flow [66].

For DAC systems with D-(+)-Mannose, the rheological behavior observed is quite similar for phases with greated or lesser amounts of solvent, i.e., an increase in viscosity to a maximum value, followed by a decrease [67]. The observed difference is that in systems with greater amounts of solvent, the maximum is reached earlier. The increase in the viscosity should characterize a deformation of the structure with, after reaching a maximum tension, will be orientated in a preferential direction, causing a decrease in the viscosity without breaking down of the cholesteric structure [67].
The flow activation free enthalpy $\left(\Delta H^{\#}\right)$ can be determined through the variation in the viscosity as a function of the temperature [68]. Systems based on KL and cholesterized by $\mathrm{D}-(+)$-Mannose showed a $\Delta H^{\#}$ decrease with an increase in the cholestericity up to a limit value of $1 \%$ molar of inductor (Fig. 5). It appears that after the movement of one micelle, the others are progressively dragged by the action of the chiral interaction. A positive $\Delta S^{\#}$ variation was observed indicating the existence of a locally transient state being less organized during the process of micellar diffusion. On the other hand, an increase in the inductor concentration leads to a decrease in the $\Delta S^{\#}$ and consequently to a system, as a whole [68].

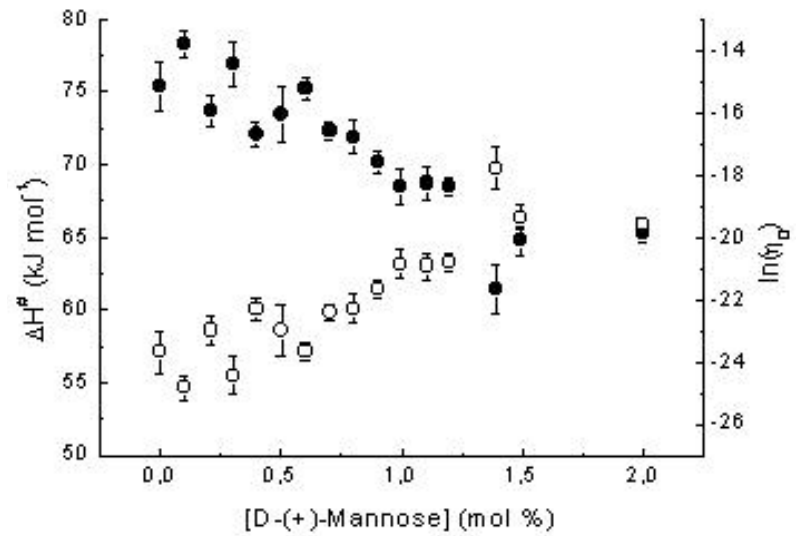

Figure 5. Activation flow enthalpy $(\bullet)$ and natural logarithmic of the pre-exponencial factor (o) as function of D- $(+)$ mannose concentration. The error bars are the standard deviations of the linear regression coefficients [68].

The DAC systems cholesterized by cholesterol show a decrease in the flow activation energy as a function of increasing inductor concentration [69]. On the other hand, when D-(+)-Mannose was used, the flow activation free enthalpy value increases. In the systems in which a hydrophobic inductor is used, the chiral forces should help in the micelle flow orientation process. It seems that, after the movement of one micelle, the others would be dragged by elastic forces, decreasing the flow activation enthalpy. When a hydrophilic inductor is used, flow activation enthalpy with increasing inductor concentration, probably because the inductor nature allows a greater interaction with the solvent, thereby acting as a barrier against the micelle flow orientation.

After twenty years of research on cholesteric lyotropic liquid crystals we can say that a complete understanding of the cholesteric structure still remains out of reach. The route to a complete understanding of the rheological properties of these systems and the factors responsible for them are just starting to be known. A lot of work still remains to be done before a level of comprehension, adequate for the complete manipulation of these systems, is reached. 


\section{Acknowledgements}

We are indebted to financial support from Fundação de Amparo à Pesquisa do Estado de São Paulo (FAPESP).

\section{References}

[1] R. Reinitzer, Monasch. Chem. 9, 421 (1888).

[2] O. Lehmann, Z. Physik. Chem. 18, 273 (1889).

[3] G. Friedel, Ann. Phys. 18, 273, (1922).

[4] H. Zocher, Trans. Faraday Soc. 29, 945 (1933).

[5] J. W. McBain, R.D. Vold, and W. J. Jameron, J. Am. Chem. Soc. 61, 30 (1939).

[6] J. W. McBain, R.D. Vold, and M. Frick, J. Phys. Chem. 44, 1013 (1940).

[7] J. W. McBain and W.W. Lee, Oil \& Soap 20, 17 (1943).

[8] J. W. McBain and W.C. Sierichs, J. Am. Chem. Soc. 25, 221 (1948).

[9] A. Saupe and G. Englert, Phys. Rev. Letters 11, 462 (1963).

[10] K.B. lawson and T.J. Flautt, J. Am. Chem. Soc. 89, 5489 (1967).

[11] L.W. Reeves, F.Y. Fujiwara, and M. Suzuki. ACS. Symp. Ser. 34, 55 (1976).

[12] B.J. Forrest and L.W. Reeves, Chem. Rev. 81(1), 1 (1981).

[13] J. Charvolin and B. Mely, ACS. Symp. Ser. 34, 48 (1976).

[14] J. Charvolin, Nuovo Cimento, 3-D, 3 (1984).

[15] J. Seeling, Quart. Rev. Byophys. 10, 353 (1977).

[16] J. Seeling and A. Seeling, Quart. Rev Byophys.13, 19 (1980).

[17] I.C.P. Smith, in NMR of Newly Accessible Nuclei, vol.2, Chemically and Biochemicaly Important Elements, Pierro Lazlo, (Ed. Academic Press, N. York, 1983).

[18] I.C.P. Smith and H.C. Jarrel, Acc. Chem. Res. 16, 266 (1983).

[19] P. Diehl and A.C. Tracey, FEBS. Lett. 59, 131 (1975).

[20] K. Radley and A. Saupe, Mol. Phys. 35, 1405 (1978).

[21] M. Acimis and L.W. Reeves, Can. J. Chem. 58, 1533 (1980).

[22] M.R. Alcantara, M.V.M.C. de Melo, V.R. Paoli, and J.A. Vanin, Coll.Int. Sci. 93, 500 (1983).

[23] M.R. Alcantara, M.V.M.C. de Melo, V.R. Paoli, and J.A. Vanin, Mol. Cryst. Liq. Cryst. 90, 335 (1983)

[24] M.R. Alcantara, M.V.M.C. de Melo, V.R. Paoli, and J.A. Vanin, Mol. Cryst. Liq. Cryst. 107, 303 (1984).

[25] M.R. Alcantara, M.V.M.C. de Melo, V.R. Paoli, and J.A. Vanin, Mol. Cryst. Liq. Cryst. 107, 359 (1984).

[26] M.R. Alcantara and J.A. Vanin, Mol. Cryst. Liq. Cryst. 102, 7 (1983).
[27] T.M.H. Aido, M.R. Alcantara, O. Felippe Jr., A.M.G. Pereira, and J.A. Vanin, Mol. Cryst. Liq. Cryst. 185, 61 (1990).

[28] T.M.H. Aido, M.R. Alcantara, O. Felippe Jr. A.M.G. Pereira, and J.A. Vanin, Mol. Cryst. Liq. Cryst. 195, 45 (1991).

[29] Y.K. Leong and D.V. Boger, J.Coll.Int. Sci. 136, 249 (1991).

[30] R.J. Hunter, Introduction to Modern Colloid Science, (Oxford University, Oxford, 1993).

[31] G.V. Vinogradov and A. Ya. Malkin, Rheology of Polymers, (MIR Publishers, Moscou, 1980).

[32] W. Richetering, J. Laüger, and R. Linemann, Langmuir, 10, 4374-4379 (1994).

[33] J. Laüger, R. Weigel, K. Berger, K. Hiltrop, and W. Richtering, J. Colloid Interface Sci. 181, 521 (1996).

[34] M.R. Alcantara and J.A. Vanin, Colloids and Surf. A 97, 151 (1995).

[35] Zs. Németh, L. Halász, J. Pálinkás, A. Bóta, and T. Horányi, Coll and Surf. A 145, 107-119 (1998).

[36] R.S. Porter, E.M. Barral, and J.F. Johnson, J. Chem. Phys. 45, 1452 (1966).

[37] D. Hantzschel, J. Schulte, S. Enders, and K. Quitzsch, PCCP Physical Chemistry Chemical Physics 5, 895904 (1999)

[38] G. Moltalvo, E. Rodenas, and M. Valiente, J. Coll. Int. Sci. 2, 232-237 (1998)

[39] D.J. Lyou and S.C. Kim, Polymer Journal 3, 279-285 (1997).

[40] V.A. Prevysh, S.A. Wang, and R.J. Spontak, Coll and Polymer Sci. 3, 284 (1997)

[41] R. Linemann, J. Laüger, G. Schmidt, and W. Richetering, Rheological Acta 5, 440 (1995).

[42] L. Walter and N. Wagner, J. Rheology 5, 1525 (1994).

[43] A.D. Rey, J. Chem. Phys. 104, 4343 (1996).

[44] C. Smyth, E.D. Kudryashov, and V. Buckin, Colloids and Surf. A 183, 517 (2001).

[45] D.V. Trukhin, O.V. Sinyachenko, V.N. Kazakov, S.V. Lylyk, A.M. Belokon, and U. Pison, Colloids and Surf B 21, 231 (2001).

[46] J.L. Ericksen, Appl. Mech Rev. 20, 1029 (1968).

[47] F.M. Leslie, Proc. R. Soc. London, Ser. A 307, 359 (1968).

[48] F.M. Leslie, Adv. Liq. Cryst. 4, 1 (1979).

[49] P.G. de Gennes and J. Prost, The Physics of Liquid Crystals, $2^{\text {nd }}$ Edition, (Oxford University Press, Claredon, 1993).

[50] E. Peuvrel and P. Navard, Liq. Cryst. 7, 95 (1990).

[51] A.D. Rey, J. Non-Newtonian Fluid Mech. 64, 207 (1996).

[52] A.D. Rey, J. Rheol. 44, 855 (2000).

[53] J. Penfold, E. Staples, A. Khan Lodhi, I. Tucker, and G.J.T. Tiddy, J. Phys. Chem. B 101, 66 (1994). 
[54] C. Gallegos, M. Nieto, C. Nieto, and J. Muñoz, Progr Colloid Polym Sci. 84, 236 (1991).

[55] J.M. Franco, J. Muñoz, and C. Gallegos, Langmuir 11, 669 (1995).

[56] T. Shikata, H. Hirata, and T. Kotaka, Langmuir 3, 1081 (1987).

[57] T. Shikata, H. Hirata, and T. Kotaka, Langmuir 4, 354 (1988).

[58] T. Shikata, H. Hirata, and T. Kotaka, Langmuir 5, 398-405 (1989).

[59] J.F.A. Soltero, J.E. Puig O. Manero, and P.C. Schulz, Langmuir 11, 3337 (1995).

[60] F. Kern, F. Lequex, R. Zana, and S.J. Candau, Langmuir 10, 1714 (1994).

[61] M.R. Alcantara, L.C.F. Dias, and J.A. Vanin, An. Assoc. Bras. Quim. 45, 47 (1996).
[62] M.R. Alcantara and L.C.F. Dias, Colloids and Surf. A 136, 155 (1998).

[63] M.R. Alcantara, A.F. de Moura, and E.G. Fernandes Jr., Mol Cryst. Liq. Cyst. 333, 69 (1999).

[64] M.R. Alcantara and A.F. de Moura, Colloids and Surf. A 175, 303 (2000).

[65] M.R. Alcantara and E. G. Fernandes Jr., Colloids and Surf. A 177, 75 (2001).

[66] M.R. Alcantara and E. G. Fernandes Jr., Mol Cryst. Liq. Cryst., in press.

[67] M.R. Alcantara and E. G. Fernandes Jr., Mol Cryst. Liq. Cryst., in press.

[68] M.R. Alcantara, A.F. de Moura, and E.G. Fernandes Jr., Mol Cryst. Liq. Cryst. 29(2), 191 (2002).

[69] E.G. Fernandes Jr., M. Sc. Dissertation, Chemistry Institute, University of S. Paulo, Brazil (2001). 